\title{
Analysis of Reform Path of University Physical Education based on Lifelong Physical Object
}

\author{
Changchun $\mathrm{Li}^{1, \mathrm{a}}$ \\ ${ }^{1}$ Xi'an International University, Xi'an, Shaanxi, 710077 \\ ${ }^{a}$ email
}

Keywords: Lifelong Physical Target, College Sports Teaching, Reform Path Analysis

\begin{abstract}
In the context of lifelong education system, lifelong physical target has been proposed, and has been practicing in practice. Thus essentially, the goal is mainly to carry out lifelong sports physical education curriculum for college students and therefore, we need to adapt their growth and health. Among them, the physical education curriculum and is not just a course, but should have some educational value, it is possible for different conditions for college students during reasonably propose appropriate teaching objectives and requirements, fully develop the overall quality of students. Therefore, this paper will focus on lifelong sports goal of reform of PE teaching path depth study.
\end{abstract}

\section{Introduction}

The main goal of lifelong sports as sports is the guiding ideology of teaching and education reform, based on the continuous development and maturity. As institutions of higher learning, the Reform of Physical Education and Lifelong Physical Education is also closely linked to the target, and between students' growth and physical activity is inseparable. Under normal circumstances, should be undertaken according to the University of Physical Education students lifelong physical education goals, the only way to enhance the effectiveness of the University of Physical Education.

\section{A Lifelong Sports Overview}

In the contemporary social context, lifelong physical education according to the needs of sports proposed, applied to university physical education, as education reform standards, can contribute to sustainable development of university sports teaching to some extent. Lifelong sports, its specific meaning can cover the following three areas: First, the core of lifelong sports is to lifelong physical exercise to ensure the effective enhance national quality and the quality of their life; secondly, always adhere to lifelong physical ideology, and actively carry out the University of Physical Education , to teach students with sports-related skills, and further develop physical activity habits, and always adhere to exercise after graduation; and finally, the key is to ensure lifelong sports on lifelong physical exercise can take the initiative, so the student initiative Training and motivation to be crucial. In general, as the main goal of the lifelong sports college sports teaching sports to teach students skills in order to develop physical activity habits, physical fitness, and enhance the quality of life for college students has important practical significance.

\section{The Present Situation of College Sports Teaching}

Teaching Philosophy and Objectives Behind Blur. Present, the domestic college physical education disadvantages are many, but the most obvious is the teaching philosophy is very backward, unable to start teaching according to the requirements of current development of specific teaching and society, so under normal circumstances, the college physical education is difficult to fully develop students' physical qualities, too Unable to carry out and implement the objectives of lifelong Physical Education. Physical Training Task think most universities, specifically refers to the learning process of students in the physical education curriculum in one or two years to obtain 
the corresponding credits. At the same time, although there are many colleges and universities have an important role in cognitive depth physical exercises of students, however, did not consider the interests of students, so students' enthusiasm is not high, it is difficult to implement the college physical education.

The Arrangement of Teaching Contents Unreasonable. Arrange for college sports programs, most of the course content in terms of arrangements and set up very unreasonable. Among them, the choice of teaching project, colleges and universities will choose the marathon or the pole vault and other projects, but with the actual situation of the students does not match. The sport requires a highly professional and physical qualities, resulting in many students are unable to complete the task, but also seriously affect their health and security risk. Thus, the above-mentioned items and Lifelong Physical Education teaching objectives do not match, therefore, reform of PE teaching is imperative.

Limited Teaching Time. Physical education stage, most universities are set by the time limit. Typically, during the freshman and sophomore, college will arrange and set the PE Teaching. However, during the junior and senior year, it will not set up physical education curriculum again. Thus, in the arrangement of College Physical Education curriculum more emphasis on credit, rather than enhance its standing in the health angle. In this case, physical education will be interrupted, too, and Lifelong Physical Training goals are not coincide, for students' lifelong physical exercise have an adverse effect.

Lack of Scientific Teaching Organization. Institutions of higher learning in the process of physical education curriculum carried out, it is easy because of the lack of organization and affect the actual teaching. The College students are generally passive recipients of knowledge, therefore, it will not take the initiative to challenge teachers to impart knowledge, to this end, but can not learn the way according to personal preference. Typically, college students based on failure to implement the growth characteristics as well as sports programs, in this case, can not be rational organization of physical activity, leading to always stay in college physical education formal level.

\section{The Teaching Reform based on Lifelong PE Target Path of PE Students}

Actively Promote the Concept of Lifelong Sports University sports teaching process carried out, we should actively promote the concept of lifelong sports and lifelong sports as a major goal, reform of $\mathrm{PE}$ teaching in full swing. In the rapid development of information technology background, the university also will enhance the level of information, therefore, physical education curriculum teaching also showing a variety of characteristics of the traditional teaching model to optimize and change, to ensure that students receive a quality physical education . College of Physical Education, the concept of lifelong physical theory which is very important, so, too, should be in the form of imparting knowledge to teach, students receive simplify difficult. At the same time, under the influence of long-term subtle, students can gradually lifelong sport philosophy. Thus, the need to carry out the University of Physical Education actively take a variety of promotional means to increase the intensity of lifelong sports publicity, especially in organized sports regularly lectures, knowledge competitions or organize sports, watching sports, etc., in this situation below, enabling students to better develop physical activity habits.

Establish and Improve the Mechanism of Physical Education Curriculum University. Affected concept of lifelong physical education, physical education is no longer a single target college students to teach sports skills, but to the students interested in sports comprehensive training, and then to develop exercise habits, so that students' physical capabilities continue to increase, and this is precisely the important goal of the University of Physical Education. In summary, the traditional teaching methods can not meet the requirements of the University of physical education of college physical education, therefore, must be a comprehensive mechanism for curriculum innovation and reform. In this process, we should pay attention to students' personality development, and respect for their interests, to create a harmonious relationship between teachers and democratization, promoting students' physical 'enthusiasm and initiative. In addition, it should take a variety of teaching methods and guided inquiry and so on, with student personality characteristics 
match. To this end, the University of Physical Education Curriculum mechanism innovation, teachers should play the role of guidance and guidelines, rational use emotional style teaching methods, and delve into more teaching methods. The lifelong sports as the main goal of teaching reform of college sports, courses should be built three of one mechanism, and thus properly guide students to engage in physical exercise, and students to develop the habit of lifelong sports.

A teaching college sports teaching is concerned, you need the full integration of health education and lifelong education, at the same time, achieve internal and external courses combine traditional and modern teaching methods combined primary and secondary materials combined with the results of the evaluation process of combining mode, more importantly, the need for teaching content appropriately adjusted, active and innovative teaching model. Wherein, in the actual teaching process, we should minimize the physical education curriculum content athletics, and on this basis, add relatively simple and relatively easy to practice, teaching content can enhance personal physical sports, especially badminton, swimming and martial arts, etc. , thereby enabling the students gradually formed the habit of lifelong physical, with lifelong sport philosophy, and always adhere to exercise. At the same time, it should be properly disposed of sport theory course add content instruction to ensure that students can better accept the sports culture and education, be changed to the traditional sports as the core teaching philosophy, and increase the share of theoretical knowledge. The content of theoretical knowledge included in the main self-help, physical exercise and mental health, etc., and then build a sound physical education system, so that theoretical knowledge in college PE teaching role into full play, college students lifelong sports consciousness.

College Physical Education to carry out, the most crucial thing is to face up to the importance of teaching evaluation. The main purpose of physical education examination is to urge students to better expand the initiative to learn and always adhere to the physical exercise. However, the physical education curriculum teaching content is the most important body motion, so that the students and the actual exercise load is critical. The most valuable is that the student through physical exercise can play a positive role in terms of physiology, in particular in many aspects of knowledge transfer, skills development and emotional promotion and so on. To this end, the University of Physical Education is carrying out the process, the need and the amount of rationality as a major sports evaluation criteria, in particular the amount of information and knowledge of exercise load and so on. On this basis, we should also pay attention to the importance of students' independent practice, and in this process, teachers need to play a supporting role, and the form of classroom tests, the students actually learning to be fully measured. Traditional sports evaluation system more attention to the students' final score, which is a healthy first serious departure from the teaching philosophy. In modern teaching, we need to evaluate the process and outcome evaluation combine together, and then to build a better evaluation system, non-discriminatory, attention to student learning process, and put their efforts into evaluation criteria which, in turn physical exercise for students create an ideal learning environment to gradually develop the habit of lifelong sports, practice teaching objectives lifelong Sports.

PE teachers also focus on teaching reform of college sports, because it is the main implementers and teaching focus on training people, so that PE teachers have to stand in the forefront of science, follow the goal of lifelong sports and training of qualified personnel through the new sports knowledge. PE teachers need to have some professional ethics and political accomplishment, a sense of responsibility and teaching experience, can accurately express and explain, through the standard operation to start teaching demonstration, effectively improve teaching effectiveness Sport University in the actual teaching, and student influence in subtle under the concept to develop lifelong sports.

\section{Conclusion}

The reform of PE teaching is complex and lengthy, requiring continue to accumulate experience in physical education development process, in-depth exploration, to achieve organic complexes with students, enrich personal knowledge of architecture and applied in practice. In summary, to carry out the University of physical education, PE teachers also need to have as a practitioner, always 
adhere to the principle of lifelong physical, active students sports consciousness, so that students consciously exercise, ultimately teaching objectives Lifelong Sports.

\section{Acknowledgements}

Project: Hongquan culture in Shaanxi private colleges and universities inheritance and promotion research.

\section{References}

[1] Wei Xiaofeng, Cha Xianfeng, Wang Wei, etc. Research Perspective of the University of Physical Education Lifelong Physical Status-a case study on Universities in Heilongjiang Province [J]. Harbin Institute of Physical Education, 2015, 33 (6): 76-79.

[2] Yi Fenggao. Lifelong Physical Education in College Physical Education Teaching Thinking [J]. Contemporary sports science and technology, 2015,5 (30): 3-4.

[3] Zhao Haijun, Yang Baoshan. Technique on the Current Status of Physical Education college sports teaching reform and innovation [J]. contemporary sports science and technology, 2016,6 (6): 75-76.

[4] Gong Shufeng. Reform of PE teaching Lifelong Sports Study [J]. Education prose one hundred hundred, 2013 (8): 5-5, 6.

[5] Zhou Peng. Physical Education Reform in China under the Perspective of thought lifelong sports [J]. Guangzhou Physical Education Institute, 2015,35 (1): 126-128.

[6] Zhu Tiefeng. Teaching Reform of Physical Education at the University of Physical Education Lifelong Perspective Thought [J]. Contemporary sports science and technology, 2016,6 (21): 67, 69. 